\title{
Bemerkungen zur Fibringlobulinfrage und Erwiderung.
}

\author{
Von \\ W. Huiskamp.
}

(Aus dem physiologischen Laboratorium der Universität Utrecht.)

(Der Redaktion zugegangen am 12. September 1905.)

Auf einige Bemerkungen Heubners ${ }^{1}$ ) zu meiner Arbeit :Zur Fibringlobulinfrage ${ }^{2}$ ) sei es mir erlaubt, in aller Kürze hier zu antworten.

Der erste Einwand Heubners betrifft die Reinheit meiner Fibrinogenlösungen. Ich habe mich mit der einfachen Angabe, daß dieselben nach Hammarsten oder auch, daß sie durch dreimalige Umfällung mit Kochsalz dargestellt wurden, begnügt. Mit Rücksicht auf die Bemerkungen Heubners scheint es mir indessen wünschenswert, das befolgte Verfahren etwas näher zu beschreiben.

Das in $1 \%$ iges Kaliumoxalat aufgefangene Pferdeblut wird möglichst bald nach der Entnahme ${ }^{1 / 8}$ Stunde zentrifugiert, wodurch die Hauptmasse der roten und weißen Blutkörperchen entfernt wird; das jetzt noch nicht vollkommen klare Plasma wird abgehebert und zur weiteren Klärung für sich noch $2-2^{1 / 2}$ Stunden zentrifugiert. Durch die vorherige Entfernung der Hauptmasse der Blutkörperchen wird erreicht, daß das Plasma möglichst kurz mit diesen Körperchen in Berührung bleibt, wodurch ein Übertritt von Ferment resp. Proferment nach Möglichkeit verhindert wird. Das jetzt vollständig klare Plasma wird mit dem gleichen Volumen gesättigter kalkfreier Kochsalzlösung versetzt, wodurch ein gallertiger Niederschlag entsteht, welcher sich somit schon äußerlich von Paraglobulin-

1) Diese Zeitschrift, Bd. XLV, S. 355.

2) Diese Zeitschrift, Bd. XLIV, S. 182.

Hoppe-Seyler's Zeitschrift f. physiol. Chemie. XLVI. 
niederschlägen unterscheidet. Die Flüssigkeit mit dem Niederschlag wird 10-15 Minuten zentrifugiert, wodurch sich zwar nicht alles Fibrinogen am Boden der Zentrifugenröhrchen ansammelt, indessen doch erreicht wird, daß der gallertige Niederschlag sich soweit zusammenzieht, daß derselbe jetzt bequem herausgenommen und ausgepreßt werden kann. Der auf diese Weise möglichst gut von Fiüssigkeit befreite Niederschlag wird mittels des anhängenden Salzes in Wasser gelöst. Es gelingt dann, ohne Verlust konzentrierte Lösungen mit nicht mehr als 2-3\% Salz darzustellen. Diese Lösung wird nochmals mit einem gleichen Volumen gesättigter Kochsalzlösung versetzt; der darauf entstandene Niederschlag ist so zähe, daß das Zentrifugieren nicht mehr nötig ist; nach einigen Augenblicken kann der Niederschlag z. B. mit einem Glasstabe soweit zusammengepreßt werden, daß derselbe zur weiteren Entfernung der Flüssigkeit aus dem Gefäß herausgenommen werden kann. Der Niederschlag wird wie oben mittels des anhängenden Salzes in Wasser gelöst, welches regelmäßig ohne Verlust stattfindet; ${ }^{1}$ ) schließlich wird nochmals mit dem gleichen Volumen gesättigter Kochsalzlösung gefällt; nach der Lösung dieses Niederschlages wird eine nicht mehr gelblich, sondern rein bläulich opalisierende Lösung erhalten, welche nach Filtration für die Versuche benutzt wurde.

Der Einwand Heubners kommt nun darauf hinaus, daß vor der ersten Fällung mit Kochsalz das Plasma von mir nicht neutralisiert wurde; aus nicht neutralisiertem Blutplasma fällt aber nach Heubner mit Kochsalz zuerst Paraglobulin und darauf erst Fibrinogen aus; ich hätte also seines Erachtens nur eine Lösung von Paraglobulin mit höchstens etwas Fibrinogen darin erhalten können.

Abgesehen davon, daß die Fällungsverhältnisse des Fibrinogens und Paraglobulins schon längst von Hammarsten zu?

1) Dieses günstige Resultat möchte ich vorzugsweise dem zur schreiben, daß die Blutkörperchen möglichst bald vom Plasma getrennt wurden und daß dieses weiterhin bis zu einer vollständig klaren Flüssigkeit zentrifugiert wurde. Wenigstens hatte ich beim nicht oder nicht genügend Befolgen dieser Maßnahmen immer Verluste zu verzeichner. 
Klarheit gebracht worden sind, kann man sich durch folgenden einfachen Versuch, welchen ich selbstverständlich nicht als neu beschreiben will, von der Unrichtigkeit der Behauptung Heubners überzeugen.

Falls die Meinung Heubners richtig wäre, müßte man erwarten, daß, nachdem das Fibrinogen durch Gerinnung aus dem Plasma entfernt worden, das Paraglobulin, bei der Halbsättigung des erhaltenen Serums mit Kochsalz ganz ebenso als beim Plasma ausfallen würde, denn die Alkaleszenz des Serums ist gewiß nicht geringer als beim Plasma. Führt man den Versuch mit Pferdeserum aus, so ergibt sich, daß die Flüssigkeit auch nach längerem Aufbewahren vollkommen klar bleibt; dasselbe gilt für Rinderserum.

Was die Ausfällung des Fibrinogens anbelangt, so liegen im Vergleich mit Pferdeplasma die Verhältnisse beim Rinderplasma insofern etwas anders, als hier durch den einfachen Zusatz eines gleichen Volumens oder selbst mehr gesättigter Kochsalzlösung nur ein sehr geringer Niederschlag entsteht, welcher jedoch, wie die Untersuchung lehrt, jedenfalls zum größten Teil aus Fibrinogen besteht. Durch vorherige Neutralisation des Plasmas läßt sich nun die Ausbeute vermehren und insofern kann die Heubnersche Modifikation als vorteilhaft betrachtet werden; allein für die Ansicht, daß man mittels der Neutralisation auch reinere Präparate als unter sonst gleichèn Umständen ohne Neutralisation erzielt, sehe ich keinen Grund. Der Einfluß der Neutralisation tritt übrigens, wie auch Mora witz ${ }^{1}$ ) bemerkt, am Rinderplasma viel deutlicher hervor als beim Pferde. Tatsächlich fällt das Pferdefibrinogen durch Halbsättigung mit Kochsalz so leicht aus, daß zur Erzielung einer guten Ausbeute weiter keine besonderen Maßnahmen nötig sind.

Der zweite Einwand Heubners betrifft die Fällung der von mir benutzten Fibrinogenlösungen mit Fluornatrium. «Auch hier, meint Heubner, «bleibt der Beweis zu erbringen, daß wirklich der ganze Niederschlag aus Fibrinogen bestand.»

Dieser Beweis wird durch die Tatsache erbracht, daß

1) P. Morawitz, Beiträge zur Kenntnis der Blutgerinnung. Dtsch. Arch. f. Klin. Medizin, Bd. LXXIX. 
Pferdeserum vollkommen klar bleibt, falls es mit dem doppelten Volumen gesättigter Fluornatriumlösung, also einer der Fällung des Fibrinogens entsprechenden Menge versetzt wird. Alle Substanzen, welche neben dem Fibrinogen im Blutplasma vorkommen, bleiben demnach beim Zusatz von $\mathrm{NaFl}$ gelöst, und diejenige Substanz, welche im Blutplasma dadurch ausfällt, ist eben das Fibrinogen; überdies muß hierbei in Betracht gezogen werden, daß die Fällung mit $\mathrm{NaFl}$ in den von mir angeführten Versuchen niemals unmittelbar aus Blutplasma geschah, sondern immer aus Fibrinogenlösungen, welche auf die oben beschriebene Weise bereitet waren. Ich möchte hier, ohne in Einzelheiten $\mathrm{zu}$ treten, noch die allgemeine Bemerkung hinzufügen, daß eine etwaige Verunreinigung des mit $\mathrm{NaFl}$ gefällten Fibrinogens die damit von mir erhaltenen Resultate in ungünstigem Sinne beeinflussen würde und daß demnach, falls meine Resultate etwa mit verunreinigtem Fibrinogen erhalten worden wären, gleich günstige Resultate a fortiori mit reinem Fibrinogen zu erzielen sein würden.

Schließlich wendet sich Heubner gegen die Art, wie ich das Fibrinogen in einigen meiner Versuche zur Koagulation gebracht habe; er findet den von mir dabei verwendeten Salzgehalt von $4 \%$ für das Erhalten richtiger Resultate zu hoch und meint, dieser Salzgehalt könne den Koagulationsgrad, das Mitausfallen usw. wesentlich modifizieren. Ich vermag nicht einzusehen, daß ein Salzgehalt von $4 \%$ in diesem Falle so besonders hoch ist. Es würde, falls überhaupt von einem wesentlichen Modifizieren des Koagulationsgrads usw. innerhalb der engen Grenzen von z. B. 1-4\% Salz die Rede sein kann, der bei einem Salzgehalt von $4 \%$ gemachte Fehler schon besonders erheblich sein müssen, bevor sich an den von mir erhaltenen Resultaten im wesentlichen etwas ändern würde. Überdies wäre erst zu beweisen, daß in diesem Falle überhaupt ein sehr geringer Salzgehalt den Vorzug verdient hätte. $\mathrm{Zu}$ bemerken ist weiter noch, daß die Versuche, um welche es sich hier handelt, $\mathrm{Pa}-$ rallelversuche waren, wobei immer dieselbe Salzkonzentration von $4 \%$ verwendet wurde und wobei es weniger auf die absoluten Mengen Fibrinogen und Fibringlobulin als vielmehr auf eine Ver- 
gleichung des Verhältnisses Fibringlobulin/Fibrinogen in den verschiedenen Fällen ankam.

Es ist zu betonen, daß, abgesehen von der Meinungsverschiedenheit, hinsichtlich der Bereitungsweise des Fibrinogens die Versuchsresultate, welche Heubner in seiner Arbeit über *die Spaltung des Fibrinogens bei der Fibringerinnung* ${ }^{1}$ ) erhalten hat, mit den Schlüssen, welche ich auf Grund der Versuchsresultate in meiner schon erwähnten Arbeit gezogen habe, vorzüglich übereinstimmen. Ich werde das im folgenden etwas näher beleuchten.

Auf Grund meiner Untersuchungen bin ich zu dem Schluß gekommen, daß das Fibrinogen im Blutplasma eine Verbindung von Fibringlobulin mit der eigentlich gerinnenden Substanz (Fibrinogen in engerem Sinne) darstellt. Es mußte dann weiter angenommen werden, daß diese Verbindung zu einem allerdings nicht sehr großen Teil hydrolytisch gespalten ist; es ergab sich noch, daß diese Spaltung, welche schon von Wasser eingeleitet wird, durch sehr geringe Mengen Alkali bedeutend gesteigert wird. Tritt im Blutplasma Gerinnung ein, so bleibt nach meiner Ansicht derjenige Teil des Fibringlobulins, welcher im Plasma hydrolytisch abgespalten war, in Lösung, während der am Fibrinogen gebundene Teil auch nach der Gerinnung am Fibrin gebunden bleibt. Das Fibrin enthält demnach an sich gebunden eine gewisse Menge Fibringlobulin, welche indessen schon durch sehr schwaches Alkali, nicht aber oder nur spurenweise z. B. durch verdünnte Kochsalzlösung abzuspalten sein würde. Tatsächlich fand nun Heubner, daß aus dem von ihm aus Blutplasma dargestellten Fibrin mit sehr verdünntem Ammoniak, nicht aber durch 10\% ige Kochsalzlösung ein Eiweißkörper ausgezogen werden konnte, welcher durch Erhitzen koaguliert und durch Sättigung mit $\mathrm{MgSO}_{4}$ ausgesalzen wurde; leider hat Heubner die weitere Natur dieses Körpers nicht feststellen können. $\mathrm{Zu}$ bemerken ist noch, daß diese Substanz nicht etwa durch das Ammoniak gelöstes Fibrin war; es zeigte sich nämlich, daß, falls das Fibrin genügend lange z. B. mit 0,02\% igem Ammoniak ausgezogen worden war, in den

1) Arch. f. exp. Pathol. u. Pharmakol., Bd. XLIX, S. 229. 
weiteren Auszügen kein Eiweiß mehr aufzufinden war, selbst nicht, falls eine viel stärkere Ammoniaklösung benutzt wurde. Auch die übrigen Versuche Heubners, wodurch gezeigt wurde, daß, wenn man dem Plasma vorher eine ganz geringe Menge Ammoniak zusetzt, der erwähnte Eiweißkörper nicht oder wenigstens nur in geringerer Menge aus dem entstandenen Koagulum mittels verdünntem Ammoniak zu erhalten ist, stehen mit meiner Anschauung sehr gut im Einklang. Die Abspaltung des Fibringlobulins ist hier durch die Anwesenheit des Ammoniaks schon im Plasma wesentlich gesteigert, welches zur Folge hat, daß sich im Fibrin nichts oder jedenfalls weniger von dieser Substanz vorfindet.

Gegen die von Schmiedeberg aufgestellte Formel für die Fibringerinnung wurde von Hammarsten der Einwand erhoben, daß nach dieser Formel die Menge des Fibringlobulins im Vergleich mit derjenigen des gebrauchten Fibrinogens viel größer sein müßte, als tatsüchlich von Hammarsten selbst gefunden worden war. Am Schlusse seiner Arbeit führt Heubner einige Versuche an, worin er die theoretische durch die Schmiedebergsche Formel geforderte Menge Fibringlobulin auch wirklich gefunden haben soll. Ein derartiger Befund, welcher darauf hinauskommt, daß die Mengen des aus dem Fibrinogen erhaltenen Fibrins und Fibringlobulins einander ungefähr gleich sind, spricht sehr für die Anschauung, daß im Plasma auf jedes Molekül Fibrinogen ein Molekül Fibringlobulin von ungefähr derselben Molekulargröße vorhanden ist; allein der Befund sagt nichts aus über die Weise, wie das Fibrinogen in Fibrin umgewandelt wird; im besonderen geht daraus keinesfalls unmittelbar hervor, (wie es Heubner auch nicht behaupten will), daß das wesentliche bei der Gerinnung in einer Abspaltung des Fibringlobulins besteht. Nach meiner Anschauung ist nicht die Abspaltung des Fibringlobulins, sondern eine Änderung im Fibrinogenmolekül selbst die Ursache der Gerinnung, und diese Anschauung kann auch ganz gut mit dem Befund, daß die Mengen des Fibringlobulins und Fibrins ${ }^{1}$ ) einander ungefähr gleich sind, in Ein-

1) Gemeint wird selbstverständlich das reine Fibrin, also ohne etwa daran gebundenes Fibringlobulin. 
klang gebracht werden. Nur will ich betonen, daß, falls etwa durch spätere Untersuchungen doch ein Überwiegen der Fibrinmenge über die Menge des Fibringlobulins festgestellt werden sollte, ein derartiger Befund sich auch mit meiner Anschauung verträgt, eben weil nach dieser Anschauung das wesentliche der Gerinnung nicht in der Abspaltung des Fibringlobulins besteht und daher die Existenz von Fibrinogenmolekülen, welche kein Fibringlobulinmolekül an sich gebunden enthalten, möglich bleibt.

Einen deutlichen Hinweis aber gegen die Meinung, daß die einfache hydrolytische Abspaltung des Fibringlobulins das Wesentliche bei der Gerinnung sei, erblicke ich in dem von mir gemachten Befund, daß sich das Fibringlobulin mittels schwach alkalischer Fluornatriumlösung wenigstens zum größten Teil aus dem Fibrinogen entfernen läßt, ohne daß der zurückbleibende Körper Fibrin ist; es resultiert vielmehr ein löslicher Körper, welcher mittels Fibrinferment durch eine bisher unbekannte Änderung in seinem Molekül in Fibrin umgewandelt werden kann. 